\title{
Diagnostiquer la proximité perçue en vente directe de produits alimentaires ${ }^{1}$
}

\author{
Version finale à paraître dans Décisions Marketing (2014), \\ janvier-mars, $\mathbf{n}^{\circ} 73$.
}

\section{Catherine HERAULT-FOURNIER}

Enseignante Chercheur, LUNAM Université, Laboratoire de Recherche En Sciences Sociales, Ecole Supérieure d'Agriculture d'Angers

Doctorante, Montpellier SupAgro, UMR 1110 MOISA

c.herault@groupe-esa.com

\section{Aurélie MERLE}

Professeur associé, Institut du Capital Client, Grenoble Ecole de Management

aurelie.merle@grenoble-em.com

\section{Anne-Hélène PRIGENT-SIMONIN}

Maître de Conférences, Laboratoire de recherche COACTIS, Université Jean Monnet

anne.helene.prigent.simonin@univ-st-etienne.fr

\footnotetext{
${ }^{1}$ Cette recherche est financée dans le cadre du programme de recherche PSDR - Liproco sur les « démarches de valorisation des produits alimentaires et activités connexes fondées sur les proximités producteurs-consommateurs ». Nous remercions vivement les trois relecteurs de Décisions Marketing pour leurs commentaires qui nous ont permis d'approfondir l'analyse et d'améliorer la qualité de cet article.
} 


\section{Diagnostiquer la proximité perçue en vente directe de produits alimentaires.}

Dans le prolongement de l'éditorial de Décisions Marketing : «La proximité est-elle une nouvelle mode du marketing ? », cet article interroge la pertinence managériale du concept de proximité en vente directe de produits alimentaires. Via des études empiriques menées dans trois circuits - l'AMAP, le Point de Vente Collectif et le marché - nous montrons qu'il est pertinent de diagnostiquer la proximité perçue par les consommateurs car elle influence positivement la confiance à l'égard des circuits étudiés et permet de les discriminer. Une fois l'intérêt du concept démontré, des recommandations sont formulées autour de deux interrogations : quels types de proximité encourager et comment développer la proximité perçue en vente directe.

Mots clés - proximité, vente directe, circuits courts alimentaires, marketing agroalimentaire, lien producteur-consommateur.

\section{Assessing the perceived proximity in direct selling of food products}

In line with the Editorial of Décisions Marketing "Is proximity a new marketing trend?", the aim of this article is to question the managerial relevance of the concept of proximity within the context of direct selling of food products. Through empirical studies in three supply chains - French CSA (AMAP), farm shops and direct selling on markets- we show the interest of assessing perceived proximity since it has a positive influence on consumers trust towards the studied supply chains and allows to discriminate them. The relevance of the concept of proximity being supported, recommendations are then emphasized on two points: which kinds of proximity to encourage and how to enhance proximity in food direct selling.

Key words - proximity, direct selling, local food, localized systems of food provision, food marketing, producer-to-consumer relationships. 


\section{Introduction}

«La proximité est-elle une nouvelle mode du marketing ?» titrait l'éditorial de Décisions Marketing de janvier 2010 (Filser et Vernette, 2010). Ce vocable de proximité connait un regain d'intérêt dans la pratique marketing, qui plus est lorsque l'on s'intéresse aux circuits de vente directe de produits alimentaires. Le développement de ces circuits, caractérisés par l'absence d'intermédiaire entre le producteur et le consommateur, a été à plusieurs reprises attribué à la plus grande «proximité » qu'ils engendraient (Holloway et alii, 2007 ; Jarosz, 2000). Les Associations pour le Maintien de l'Agriculture Paysanne (AMAP), créées il y a une dizaine d'années, attirent à titre d'exemple plus de 200000 consommateurs en 2010 avec un chiffre d'affaires estimé à 36 millions d'euros et un taux de croissance qui flirte dans certains départements autour des $50 \%$ chaque année. Dans le prolongement de l'éditorial précité (Filser et Vernette, 2010), l'objectif de cet article est de confirmer la pertinence managériale du concept de proximité dans le cadre de la vente directe de produits alimentaires, en l'étudiant dans trois formats : le Point de Vente Collectif (PVC), l'AMAP et la vente directe en marché. Il élargit la portée et complète les travaux de Hérault-Fournier, Merle et Prigent-Simonin (2012) qui s'intéressent uniquement aux PVC. Nous apportons ici des éléments de réponse à deux questions (objectifs et méthodologie synthétisés dans le tableau 1):

1- Que signifie être proche en vente directe ? Une étude qualitative auprès de 35 acheteurs, couplée à la littérature sur le sujet, nous permet de développer les dimensions de la proximité perçue - proximité d'accès, identitaire, relationnelle et de processus - en les analysant dans ces trois formats de vente directe.

2- Est-il pertinent pour des professionnels en vente directe de diagnostiquer la proximité perçue de leur mode de commercialisation? Pour démontrer scientifiquement l'intérêt managérial du concept, nous analysons son pouvoir prédictif et discriminant. Autrement dit, nous cherchons à : (2a) confirmer si la proximité perçue influence une variable considérée comme importante dans la création et le maintien d'une relation : la confiance et $(2 b)$ montrer qu'il est possible de différencier des modes de commercialisation en vente directe en analysant la proximité perçue par les consommateurs. Via une étude quantitative auprès de 579 acheteurs, dans ces trois circuits (encadré 1), nous validons que la proximité influence positivement la confiance à l'égard du circuit de vente directe et précisons la place de chaque dimension dans le modèle. L'analyse comparée des scores de proximité perçue dans ces 
circuits démontre par ailleurs le pouvoir discriminant du concept. Les analyses réalisées permettent au final de formuler des recommandations managériales autour de deux interrogations: quels types de proximité encourager et comment développer la proximité perçue en vente directe. 


\section{Encadré 1 : Trois formats de vente directe observés}

La vente directe est un circuit de distribution sans intermédiaire entre le consommateur et le producteur. Ce dernier assure lui-même la production et la distribution de ses marchandises. Les études qualitative et quantitative menées portent sur trois formes de vente directe de produits alimentaires correspondant à des initiatives aux modalités pratiques différentes, qui ont toutes connu un fort développement : l'AMAP, le Point de Vente Collectif et la vente directe sur les marchés.

\begin{tabular}{|c|c|c|}
\hline & Principe & $\begin{array}{c}\text { Quelques dates } \\
\text { et chiffres }\end{array}$ \\
\hline 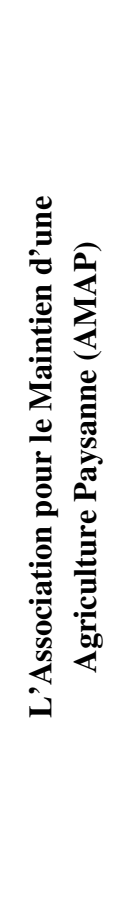 & $\begin{array}{l}\text { Une AMAP est un partenariat entre un groupe de } \\
\text { consommateurs et un ou plusieurs agriculteurs, autour } \\
\text { d'engagements mutuels. Les producteurs s'engagent à fournir } \\
\text { des produits divers et de qualité et les consommateurs } \\
\text { s'engagent à payer à l'avance et à venir récupérer leurs } \\
\text { «paniers » de produits sur un lieu de distribution unique et à un } \\
\text { horaire déterminé. } \\
\text { La charte des AMAP, qui définit les valeurs et engagements de } \\
\text { ces associations, s'appuie sur } 18 \text { principes (www.reseau- } \\
\text { amap.org/docs/chartedesamap.PDF) et insiste notamment sur : } \\
\text { (a) La solidarité entre les acteurs de l'échange et une } \\
\text { participation active des consommateurs, (b) Des exploitations à } \\
\text { taille humaine avec des pratiques respectueuses de } \\
\text { l'environnement et (c) Une transparence dans les actes d'achat } \\
\text { et de production. } \\
\text { En 2010, le mouvement Inter-Régional des AMAP } \\
\text { (MIRAMAP) est créé avec pour objet de «renforcer la cohésion } \\
\text { des AMAP à travers le partage d'une éthique commune, de } \\
\text { mutualiser les expériences et les pratiques et d'assurer la } \\
\text { représentation et la mise en valeur des AMAP au niveau } \\
\text { national» (www.miramap.org). }\end{array}$ & $\begin{array}{l}\text { - 2001: Création de la } \\
1^{\text {ère }} \text { AMAP en France } \\
\text { - } 2003 \text { : Dépôt de la } \\
\text { marque AMAP à l'INPI } \\
\text { - } 2012 \text { : } \\
\text { Plus de } 1600 \text { AMAP en } \\
\text { France : } 214 \text { en Rhône- } \\
\text { Alpes et } 152 \text { en } \\
\text { Provence-Alpes- Côte } \\
\text { d'Azur } \\
66000 \text { familles, } 270000 \\
\text { consommateurs } \\
\text { Chiffre d'affaires estimé } \\
\text { à } 48 \text { millions d'euros } \\
\text { Présence dans plus de } 20 \\
\text { pays sous différentes } \\
\text { dénominations }\end{array}$ \\
\hline 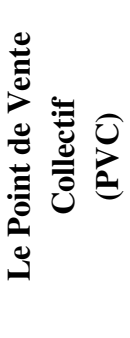 & $\begin{array}{l}\text { Le Point de Vente Collectif est un magasin où les producteurs } \\
\text { vendent eux-mêmes, en direct, les produits issus de leurs } \\
\text { exploitations. Il regroupe plusieurs producteurs d'exploitations } \\
\text { distinctes qui gèrent collégialement le point de vente. L'offre est } \\
\text { parfois complétée par celle d'autres agriculteurs proposant leurs } \\
\text { produits en dépôt-vente. }\end{array}$ & $\begin{array}{l}\text { - } 1978 \text { : Création du } 1^{\mathrm{er}} \\
\text { PVC dans le Rhône } \\
\text { - } 2010 \text { : } 69 \text { PVC en } \\
\text { Rhône-Alpes } \\
\text { - Croissance de } 165 \% \\
\text { entre } 2000 \text { et } 2010 \\
\text { - } 150 \text { à } 200 \text { PVC } \\
\text { aujourd'hui en France }\end{array}$ \\
\hline 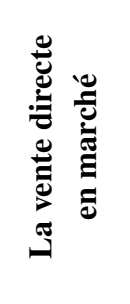 & $\begin{array}{l}\text { Un marché est composé la plupart du temps de commerçants et } \\
\text { d'artisans, alimentaires ou non, auxquels s'ajoutent des } \\
\text { producteurs. La vente directe correspond à la vente par ces } \\
\text { agriculteurs des produits issus de leurs exploitations. }\end{array}$ & $\begin{array}{l}\text { - De } 65 \text { marchés en } \\
2001 \text { à } 120 \text { en } 2009 \text { dans } \\
\text { le Rhône } \\
\text { - Premier lieu d'achat de } \\
\text { produits alimentaires } \\
\text { locaux }\end{array}$ \\
\hline
\end{tabular}

Source : http://liproco-circuits-courts.com / 
Tableau 1

Méthodologie des études empiriques ${ }^{2}$

Objectif 1 :

Comprendre ce que représente la proximité pour les acheteurs en vente directe

Objectif 2 :

Analyser l'intérêt managérial du concept de proximité en vente directe et formuler des recommandations

2b. Analyser son pouvoir discriminant : la proximité permet-elle de différencier des formats de vente

directe?

2a. Analyser son pouvoir prédictif : la proximité a-t-elle une influence sur la confiance à l'égard d'un circuit de vente directe?

\section{METHODOLOGIE}

Méthode de collecte des données

35 acheteurs dans trois circuits de vente directe : 3 AMAP ( $n=$

Taille d'échantillon

et mode

d'administration

$9), 3$ points de vente collectifs $(n=18)$ et 3 marchés $(n=8)$

Entretiens semi-directifs
Enquete par questionnaires

579 acheteurs interrogés dans trois circuits de vente directe : plus de 50 AMAP $(n=267), 2$ points de vente collectifs $(n=184)$ et 6 marchés $(n=128)$.

Administration sur les lieux de vente pour les PVC et marchés et couplage d'une administration sur les lieux de vente et par Internet pour les AMAP

Chaque acheteur est interrogé uniquement sur ses pratiques concernant un lieu de vente

Caractéristiques Profils hétérogènes en termes d'âge (25-78 ans), de sexe (12

des échantillons

hommes et 23 femmes), CSP et fréquence d'achat
$76 \%$ de femmes, $67 \%$ de plus de 40 ans, surreprésentation des cadres-professions intellectuelles supérieures (36\%) et des retraités (29\%). 75\% d'acheteurs hebdomadaires

Questionnaire structuré en 4 parties mesurant : 1- les proximités perçues (échelles d'accord en 5 points), 2- les comportements d'achats, 3- la confiance, 4- les Guide d'entretien structuré autour de deux thèmes : le rapport caractéristiques socio-démographiques

des consommateurs au magasin et aux produits et leur rapport Items de proximité perçue adaptés de Hérault-Fournier et al. (2012). Confiance à aux producteurs

l'égard du circuit de vente directe adaptée de l'échelle en 5 points de Gurviez et Korchia (2002)

Les qualités psychométriques des échelles ont été vérifiées (Annexe 2)

Méthode d'analyse des données

Mode d'analyse Analyse de contenu thématique à l'aide de NVivo 8. Doublecodage sur plusieurs entretiens.
Méthode d'équations structurelles sur la matrice des variances-covariances entre toutes les indicatrices, AMOS 17
ANCOVA (covariants : âge, budget, ancienneté et fréquence de la relation au circuit)

\footnotetext{
${ }^{2}$ Le détail des caractéristiques des échantillons qualitatifs et quantitatifs, ainsi que les outils de collecte (guide d'entretien et questionnaire) sont disponibles sur demande auprès des 4 auteurs.
} 


\section{Qu'est-ce que la proximité perçue par des acheteurs en vente directe?}

Largement mobilisé en économie, le concept de proximité est utilisé pour étudier les modalités de coordination entre les acteurs économiques (encadré 2).

\section{Encadré 2 : Le concept de proximité en économie, fondements théoriques}

En économie, l'analyse de la proximité se formalise au sein d'un courant de pensée appelé «Dynamiques de proximité », cherchant à qualifier les relations entre ensembles (Bellet, Kirat et Largeron, 1998). Si différentes typologies des formes de proximité ont pu être proposées, un consensus existe autour de la distinction entre une proximité géographique et organisationnelle. La première se rapporte à la distance physique perçue entre les acteurs et est relative au temps et aux coûts de transport (Bouba-Olga et Grossetti, 2008 ; Rallet et Torre, 2005). La proximité organisationnelle concerne «la capacité qu'offre une organisation de faire interagir ses membres, l'organisation désignant ici tout ensemble structuré de relations, formelles ou informelles » (Rallet et Torre, 2005). Deux sous-dimensions de cette proximité sont identifiées (Gilly et Torre, 2000) : la proximité de similitude et la proximité d'appartenance. La logique de similitude correspond à un lien créé par le partage d'un même système de représentations et de croyances alors que la logique d'appartenance est liée à l'interaction entre acteurs, facilitée par des règles communes et des routines. Cette dernière forme de proximité a été plus récemment qualifiée de proximité relationnelle par Bouba-Olga et Grossetti (2008) dans le cas où les acteurs appartiennent à des entités différentes.

En comportement du consommateur, ce concept a également été utilisé pour étudier les liens entre des individus et une marque, un fournisseur de service ou une enseigne de distribution (Dampérat, 2006, Bergadaà et Del Bucchia, 2009). Parmi ces travaux, celui de Bergadaà et Del Bucchia (2009) utilise la proximité pour étudier les relations entre un client et une enseigne de distribution alimentaire et offre en cela un cadre d'analyse intéressant. Il fait émerger cinq dimensions de la proximité, trois d'entre elles étant proches de celles identifiées dans les théories économiques : la proximité d'accès est comparable à la proximité géographique. La proximité identitaire, c'est à dire le partage 
de valeurs entre deux acteurs, rejoint les formes de proximité dites de similitude. Enfin, la proximité relationnelle revêt le même nom qu'en économie de la proximité et correspond aux relations directes entre le consommateur et le personnel en contact.

Les deux autres dimensions - la proximité de processus et fonctionnelle- semblent plus spécifiques à l'analyse des relations entre un client et un point de vente ainsi qu'à l'achat de produits alimentaires. La première renvoie à la connaissance du fonctionnement interne du magasin, de l'origine des produits. La seconde fait référence à la recherche d'efficacité dans l'acte de magasinage : ne pas perdre de temps, trouver facilement les produits, et correspond donc à la définition de la valeur utilitaire.

Nous utilisons ici le concept de proximité pour étudier la relation entre un individu et un circuit de vente directe. Interrogés qualitativement (tableau 1) sur leur rapport au point de vente et aux producteurs dans différents circuits (encadré 1) les acheteurs en vente directe évoquent quatre des dimensions préalablement identifiées: les proximités d'accès, relationnelle, identitaire et de processus. Les définitions de chacune des dimensions ainsi que des verbatim dans chacun des circuits sont présentés en Annexe 1.

\section{Proximité d'accès : se rendre facilement sur le point de vente}

Les consommateurs achetant leurs produits en vente directe définissent la proximité à l'égard du circuit par une proximité spatiale, classiquement appréhendée en économie au travers de distances physiques, de temps de transport, de coûts de transport, de coûts de communication (encadré 2) : "moi, c'était le côté distance, que je puisse y aller à pied» (Amap, Femme, 39 ans, documentaliste), «il est près de chez nous, (...) il est facile d'accès » (Marché, Homme, 49 ans, enseignant-chercheur). Plus qu'une question de distance, c'est aussi l'accessibilité perçue au marché, au PVC ou au point de distribution de l'AMAP qui est importante : "Je trouve qu'il est bien situé parce que pour amener mes enfants à différents endroits j'y passe »(PVC, Femme, 43 ans, enseignante). Une enquête conduite par le CRIOC (Vandercammen, 2010) souligne le rôle essentiel de la proximité géographique dans le choix d'un magasin alimentaire (premier critère cité par 39\% des répondants), loin devant le prix (16\%) ou la qualité $(12 \%)$. Ce critère d'accessibilité depuis le domicile ou le lieu de travail, apparaît également pertinent dans la définition de la proximité en vente directe. Il revêt même 
une dimension supplémentaire, puisque l'accessibilité au point de vente permet également une accessibilité au producteur, présent sur le lieu de vente.

\section{Proximité relationnelle : échanger avec le producteur}

En vente directe, le producteur est au cœur du système et la possibilité d'échanges répétés sur le lieu de distribution et/ou de production est un élément clé de différenciation par rapport à des systèmes concurrents. Le lien direct créé avec «son » producteur est de nombreuses fois cité par les acheteurs pour qualifier la proximité au circuit de vente. Cette proximité, dite relationnelle, évoque principalement des échanges d'informations sur les produits vendus, les modes de production et de préparation. Néanmoins, des relations d'amitié se tissent parfois avec les producteurs, certains abordant des sujets plus personnels : «c'est un petit peu familier les rapports et puis (...) on se connait bien » (Marché, Femme, 48 ans, gestionnaire de laboratoire), «c'est une relation conviviale, cordiale, amicale. Enfin amicale, (...) l'adjectif est peut-être un peu fort, cordiale en tout cas » (Marché, Femme, 51 ans, technicienne). On retrouve ainsi la distinction entre des interactions fonctionnelles, directement liées à la tâche à accomplir, et des interactions sociales (Bendapudi et Berry, 1997 ; Dampérat, 2006).

\section{Proximité identitaire : partager des valeurs}

La proximité est également abordée par les acheteurs en vente directe comme "une philosophie », « un état d'esprit», «des valeurs communes». Articulées autour de la volonté d'inscrire ses pratiques dans une perspective plus durable, les valeurs défendues dans l'achat direct aux producteurs sont plurielles et renvoient aux deux dimensions de la consommation responsable, sociale et environnementale (Merle et Piotrowski, 2012) : faire évoluer les modèles de production et de consommation dominants (Brunori, 2007), soutenir les petits producteurs locaux (Seyfang, 2006), protéger l'environnement (Dubuisson-Quellier et Lamine, 2004) ou bien encore préserver la biodiversité et le paysage (Sirieix, Grolleau et Schaer, 2008). «La proximité n'est pas seulement avec les producteurs. Mais on se rend bien compte que la plupart des consommateurs, on se retrouve très souvent dans différentes manifestations. On voit bien que la philosophie est quand même partagée sur beaucoup de plans et beaucoup de points » (Amap, Femme, 31 ans, professeur). Le partage de ces valeurs avec les producteurs mais aussi 
entre consommateurs renvoie à la notion de proximité identitaire de Bergadaà et Del Bucchia (2009).

\section{Proximité de processus : connaître «les manières de faire »}

Enfin, être proche en vente directe est également défini par les acheteurs comme le fait de connaître les manières de produire, de transformer et de distribuer des producteurs. Cette proximité dite de processus renvoie donc au degré de proximité perçue par rapport au fonctionnement interne du système de vente directe. Etre informés « de ce qu'ils ne voient pas » semble important dans la définition de la proximité en vente directe : "être proches c'est-à-dire, on a besoin de savoir d'où ça vient, savoir comment il fait, sa façon de procéder, sa technique » (Amap, homme, vendeur, 53 ans). Ceci apporterait aux consommateurs une forte garantie sur les produits vendus (Bergadaà et Del Bucchia, 2009). «C'est cher mais on a la garantie que les produits sont bons, que c'est des petits producteurs qui ne mettent pas trop de pesticides dans la terre, du moins j'espère » (PVC, Femme, 56 ans, retraitée).

Les entretiens approfondis avec des acheteurs en AMAP, PVC et en vente directe sur les marchés, couplés à une analyse de la littérature nous permettent donc de confirmer quatre dimensions de la proximité : d'accès, identitaire, relationnelle et de processus. Sur cette base, un outil de diagnostic quantitatif de la proximité perçue à un circuit de vente directe est proposé (Qualités psychométriques en annexe 2). Son utilisation passe par un prérequis : confirmer l'intérêt managérial que peut avoir un circuit de vente directe à diagnostiquer la proximité perçue.

\section{Quel est l'intérêt de diagnostiquer la proximité perçue ?}

Le concept de proximité est relativement nouveau et donne lieu à des interrogations sur son utilité (Filser et Vernette, 2010). Nous démontrons qu'il est pertinent à prendre en compte pour des manageurs en vente directe : 1- parce qu'il influence une autre variable considérée comme importante en marketing relationnel : la confiance, 2- parce qu'il permet de distinguer des formats de vente directe et, de ce fait, qu'il peut constituer un élément de différenciation. Pour ce faire, une étude quantitative (tableau 1) est réalisée auprès de 579 acheteurs dans trois circuits (encadré 1). 


\section{Quel pouvoir prédictif? L'influence de la proximité sur la confiance}

Est-il approprié de travailler sur la proximité perçue pour construire des relations de qualité avec ses clients en vente directe ? Répondre à cette interrogation nécessite de savoir si la proximité impacte d'autres variables clés en marketing relationnel. La confiance et l'engagement sont les deux construits les plus souvent étudiés, sans que les travaux n'attestent de la primauté de l'un sur l'autre (Palmatier et alii, 2006).

Nous faisons ici le choix de tester le pouvoir prédictif des quatre formes de proximité sur la confiance à l'égard du circuit, définie, dans la lignée de Morgan et Hunt (1994), comme la croyance dans la crédibilité et l'intégrité du circuit de vente directe dans lequel le consommateur réalise ses achats. Trois raisons justifient le choix de la confiance. L'hypothèse d'une influence positive de la proximité sur cette variable a été émise à plusieurs reprises (Bergadaà et Del Bucchia, 2009 ; Bouba-Olga et Grossetti, 2008 ; Gilly et Torre, 2000). Les travaux conduits sur les points de vente collectifs (Hérault-Fournier, Prigent-Simonin et Merle, 2012) attestent que, dans ce contexte, la proximité identitaire, de processus et relationnelle ont une influence positive directe sur la confiance perçue à l'égard du circuit. Par ailleurs, la confiance est considérée comme un atout important pour la satisfaction et la fidélisation du consommateur notamment dans le secteur agroalimentaire (Sirieix et Dubois, 1999). Enfin, elle est souvent modélisée comme un antécédent de l'engagement (Morgan et Hunt, 1994).

La figure 1 récapitule les résultats du modèle structurel soumis aux données empiriques. L'étude qualitative des formes de proximité dans les trois circuits étudiés a montré que les proximités identitaire et de processus peuvent être construites sur la base de la relation directe producteur-consommateur. C'est pourquoi le lien de la proximité relationnelle vers ces deux autres dimensions est ici intégré au modèle. 


\section{Figure 1}

L'influence des formes de proximité perçue sur la confiance à l'égard du circuit de vente directe alimentaire

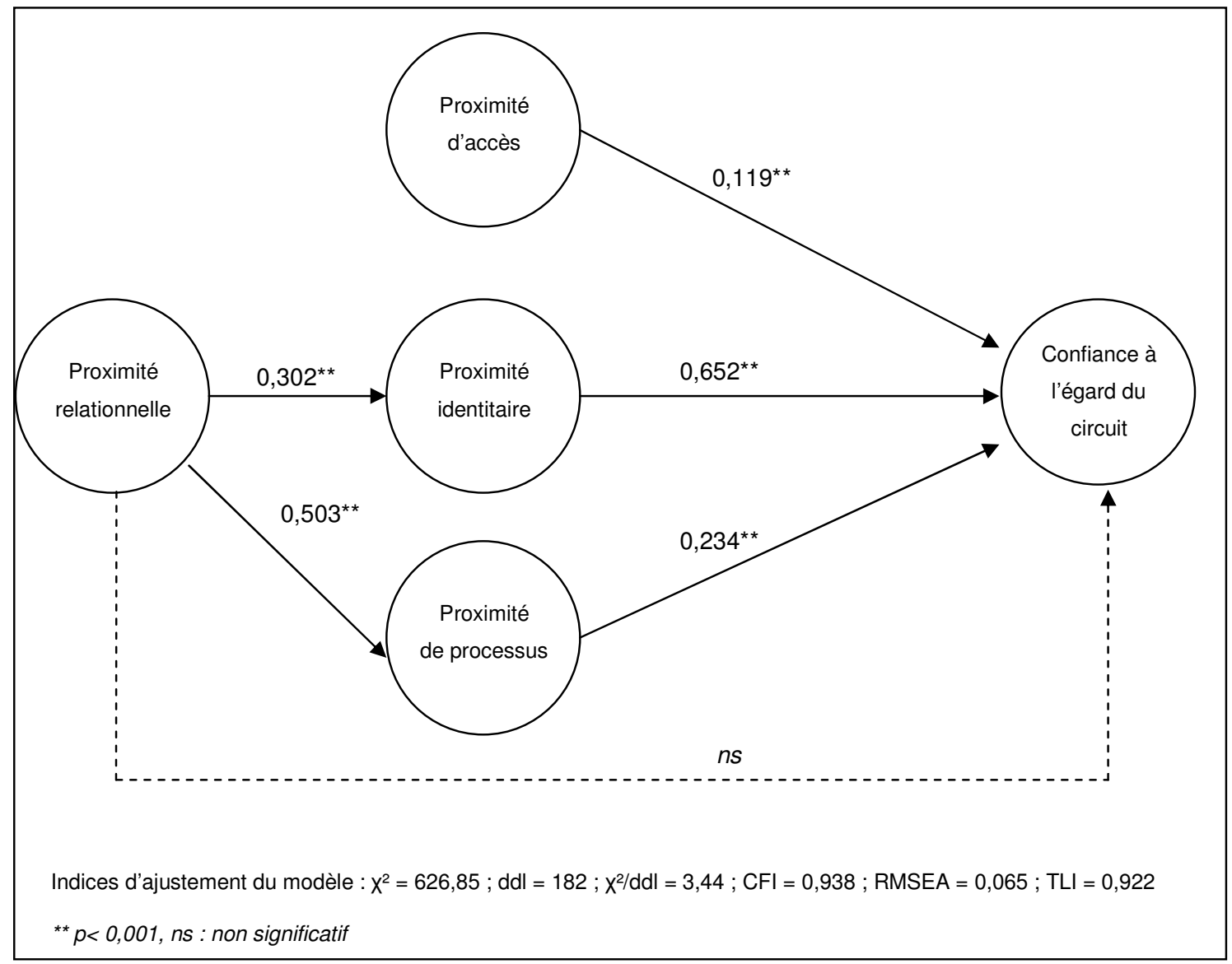

La confiance à l'égard du circuit de vente directe est influencée positivement et directement, par ordre croissant, par : la proximité identitaire $(\lambda=0,652 ; p<0,001)$, la proximité de processus $(\lambda=0,234 ; p<0,001)$, et faiblement par la proximité d'accès $(\lambda$ $=0,119 ; p<0,001)$. Au contraire, la proximité relationnelle n'influence pas directement la confiance à l'égard du circuit. Elle y participe néanmoins via son rôle dans la construction de proximité identitaire $(\lambda=0,302 ; p<0,001)$ et de proximité de processus $(\lambda=0,503 ; p<0,001)$. Au final, l'effet total de la proximité relationnelle sur la confiance est de 0,331 .

Les dimensions de la proximité identifiées possèdent donc un pouvoir explicatif intéressant: elles permettent de restituer $56 \%$ de la variance de la confiance, confirmant l'intérêt prédictif de l'outil de diagnostic. 
Par ailleurs, si le rôle de la proximité relationnelle dans la création de confiance est largement mis en avant dans les circuits de vente directe de produits alimentaires, nos résultats (a) précisent que le processus par lequel elle peut impacter la confiance est indirect et (b) modèrent les propos sur la force de ce lien. En effet, l'intensité de l'échange direct avec les producteurs n'influence pas directement la confiance : elle la favorise à travers la création de valeurs partagées (proximité identitaire) et la connaissance des modes de production et règles du circuit (proximité de processus). D'autres moyens que la relation directe avec le producteur, développés dans les recommandations (encadré 5 et tableau 2), semblent être à même d'accroitre ces deux proximités compte tenu des pourcentages de variance expliquée (respectivement 9,12\% et $25,3 \%$ expliquées par la proximité relationnelle).

\section{Quel pouvoir discriminant? Une proximité perçue différente selon les formats de vente}

Un format de vente peut-il se différencier via la proximité qu'il suscite ? L'étude réalisée permet de répondre par l'affirmative. Il est possible de distinguer les trois modes de commercialisation par les proximités qu'ils engendrent, à l'exception de la proximité d'accès. En effet, les scores de proximité identitaire, de processus et relationnelle y sont significativement différents $\left(\mathrm{F}_{\mathrm{PI}}(2,473)=36,26 ; p<0,001, \mathrm{~F}_{\mathrm{PR}}(2\right.$, $\left.471)=20,10 ; p<0,001, \mathrm{~F}_{\mathrm{PP}}(2,474)=62,49 ; p<0,001\right)$. La proximité d'accès est quant à elle évaluée de la même manière quel que soit le circuit $\left(\mathrm{F}_{\mathrm{PA}}(2,474)=1,46 ; p\right.$ $>0,10)$.

L'analyse plus fine des scores (figure 2) et des facteurs influant sur ces scores permet d'identifier des pistes d'actions vectrices de proximité. 


\section{Figure 2}

Scores de proximité perçue en fonction des modalités de vente directe

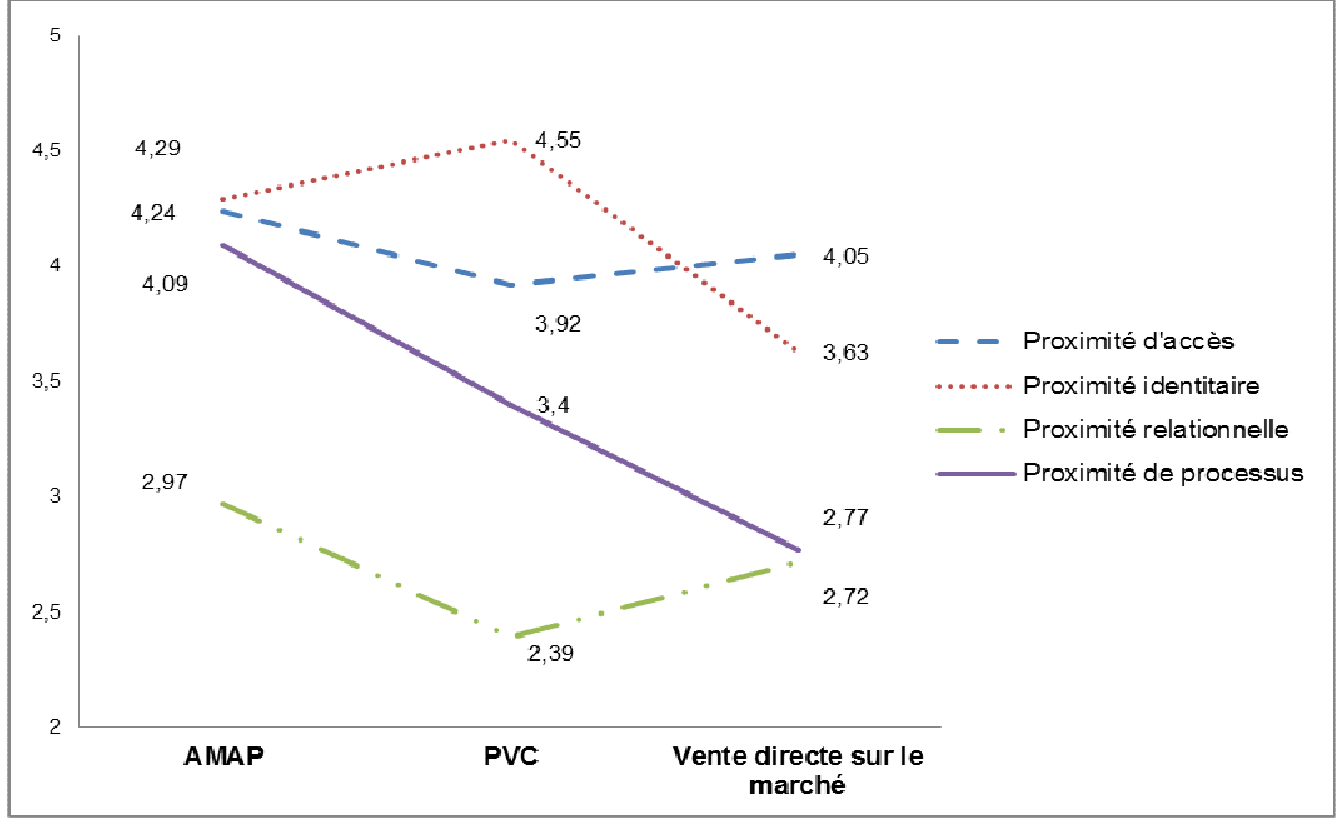

\section{- Les atouts des PVC pour une proximité identitaire forte}

«Etre proche » en vente directe correspond avant tout au fait de partager des valeurs relatives aux façons de produire et de consommer, comme le montrent les scores de proximité identitaires relativement élevés (figure 2).

La proximité identitaire constitue un fort vecteur de différenciation entre les modalités de vente directe. Son score est significativement plus élevé dans les PVC (estimation de contraste marché-PVC $=-0,893 ; p<0,001)$ et les AMAP (estimation de contraste marché$A M A P=-0,670 ; p<0,001)$. Dans ces deux circuits, les producteurs sont engagés dans une dynamique collective, soit au niveau d'une initiative en particulier (exemple : des producteurs se regroupent pour créer un point de vente collectif ou participer à une AMAP), soit à un niveau plus global fédérant plusieurs initiatives autour d'un même concept de distribution (exemple : le réseau MIRAMAP présenté encadré 1 ou Terre d'Envies en encadré 3). Cette dynamique collective favorise la proximité identitaire en s'appuyant principalement sur deux leviers : la formalisation des valeurs et la capacité des producteurs à les communiquer.

Formaliser ses valeurs

La charte qui définit les principes fondateurs de l'AMAP permet de fédérer les producteurs et les consommateurs autour d'une éthique commune (voir encadré 1). La 
volonté de produire et de consommer « autrement » sous-tend l'existence même de cette forme de vente directe. Les PVC, du fait de leur fonctionnement collégial, amènent également les producteurs à préciser ensemble les valeurs sur lesquelles ils souhaitent s'appuyer. La dynamique collective est cette fois-ci intra-magasin : les valeurs peuvent diverger d'un PVC à l'autre, même si l'association Terre d'Envies contribue à initier une dynamique régionale (encadré 3). Au contraire, les stands de producteurs au sein d'un même marché se caractérisent souvent par une grande diversité et un manque de formalisation des valeurs tant au niveau individuel que collectif.

\section{Encadré 3 : Créer de la proximité par la mise en réseau. L'exemple de Terre d'Envies en Rhône-Alpes}

Date de création : 1990

Nombre de salariés : 2,5

Statut : Association loi 1901

Nombre d'adhérents : 26

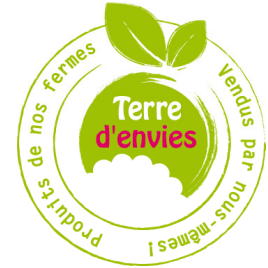

Charte Terre d'Envies: elle met en évidence les grands principes de fonctionnement d'un PVC, notamment: (a) la mise en avant de la présence du producteur, qui permet de différencier le PVC des autres commerces de proximité et des revendeurs (b) la spécification des bonnes pratiques par production incitant à « une agriculture à dimension humaine, dans le respect de la nature et de l'animal».

Questions posées à Carine Montet, animatrice Terre d'Envies:

D.M. : Quelles sont les missions de l'association Terres d'Envies?

«On peut distinguer 4 grandes missions :

- Assurer la défense et la reconnaissance des points de vente collectifs afin qu'ils soient considérés comme une forme à part entière de vente directe.

- Accompagner les membres de l'association pour faciliter le fonctionnement de leur point de vente collectif et favoriser leur développement économique

- Etre source d'informations pour les points de vente

- Assurer la promotion des points de vente collectifs via la création d'une marque collective Terre d'Envies associée à une nouvelle identité visuelle»

D.M. : Pourquoi avoir souhaité créer cette nouvelle identité ?

«Chaque point de vente veut garder son identité. En plus ils sont vraiment très différents les uns des autres. Il faut donc veiller à ce que chacun préserve son identité, mais qu'on arrive à faire en sorte que les consommateurs comprennent qu'ils font partie d'un réseau et qu'ils ont des valeurs communes avec d'autres magasins sur la région ».

D.M. : Quel est le noyau dur sur lequel vous avez donc communiqué ?

«La présence du producteur à la vente et la qualité des produits. Ce sont les deux éléments que les clients des points de vente ont bien intégré et qui correspondent à des valeurs fondamentales pour eux, c'est ce pourquoi ils viennent ». 


\section{Communiquer sur ses valeurs}

Malgré des valeurs très fortement ancrées, les AMAP possèdent un score de proximité identitaire moins élevé que les PVC (estimation de contraste ${ }_{A M A P-P V C}=-0,222 ; p<$ 0,05). Ces derniers possèdent en effet plusieurs atouts : un local fixe permettant de développer de la publicité sur le lieu de vente, un budget collectif réservé à la communication, la présence éventuelle de salariés. Au contraire, la gestion bénévole de l'AMAP par les consommateurs peut expliquer le peu d'outils de communication utilisés pour relayer les valeurs formalisées au niveau du réseau. Sur les marchés, les producteurs développent quant à eux très peu de supports d'information, entretenant la confusion avec les revendeurs, et rendant difficile l'émergence d'une proximité identitaire forte.

\section{- La capacité des AMAP à générer une proximité de processus plus forte}

Un peu moins élevée que la proximité identitaire, l'évaluation de la proximité de processus montre que les acheteurs interrogés ont une vision relativement claire des manières de faire sous jacentes aux modalités de vente directe. Cette dimension de la proximité est toutefois beaucoup plus forte en AMAP que sur les marchés (estimation de contraste $\left.{ }_{A M A P-m a r c h e ́}=1,51 ; p<0,001\right)$ et en PVC (estimation de contraste ${ }_{A M A P-P V C}=$ $0,793 ; p<0,001)$. La encore, deux éléments peuvent expliquer ces écarts : le degré de formalisation des principes d'action et la capacité à les communiquer.

\section{Formaliser ses principes d'action}

La charte des AMAP implique des engagements forts de la part des producteurs sur leur manière de produire, de s'approvisionner et de fixer les prix, donnant au consommateur des indications claires, au niveau national, sur le processus (encadré 1). Par ailleurs, si le label Agriculture Biologique n'est pas obligatoire, il est toutefois vivement encouragé dans certaines régions, comme en Ile de France où les producteurs doivent se convertir dans les trois années qui suivent leur installation en AMAP. De son côté, la charte des PVC proposée en Rhône-Alpes fournit un cadre général pour le bon fonctionnement du point de vente (encadré 3). Elle exige une transparence sur les modes de production mais n'impose pas de pratiques uniformisées, expliquant sans doute en partie le score de proximité de processus plus faible. 


\section{Communiquer sur ses principes d'action}

La communication sur ses principes d'action joue également un rôle clé. Elle peut être réalisée par les producteurs ou via une co-construction avec le consommateur.

Sur les marchés, les producteurs font parfois état des engagements pris concernant leurs pratiques à travers la labellisation de leur mode de production. En dehors de ce cadre, la proximité de processus se construit majoritairement à travers l'échange verbal avec le consommateur. Etroitement liés à la volonté et la disponibilité des acteurs, ces échanges apparaissent très variables du point de vue de leur intensité comme du point de vue de la nature des informations échangées. Certains PVC mettent à disposition des clients des supports écrits, appelés «cahiers de transparence», permettant de mieux comprendre les modes de production, la transformation ou les produits de chacun.

Le fort score en AMAP semble pour sa part s'expliquer en partie par la co-construction $\mathrm{du}$ processus. Le consommateur peut s'engager activement et participer tant aux activités de production, qu'à la fixation des prix et à la distribution des paniers, renforçant son contrôle perçu. Ce rapprochement de l'activité de production crée nécessairement une plus grande connaissance du fonctionnement interne du circuit (encadré 4). La participation des consommateurs, inhérente au fonctionnement des AMAP, est parfois possible dans les PVC et, de manière plus marginale, sur les marchés, via des visites d'exploitations.

\section{Encadré 4 : Les AMAP, une proximité renforcée par la co-construction}

En 2010, dans les AMAP du Maine et Loire, les Amapiens se sont engagés auprès des producteurs :

- L'éleveur de chèvres de l'AMAP des Goganes lance un chantier «bardage ». Les chèvres doivent emménager dans une nouvelle installation rapidement sans que le producteur n'ait le temps de réaliser le bardage du nouvel atelier. Avec l'aide d'une dizaine d'amapiens, le chantier est finalisé en une demi-journée.

- L'éleveur laitier, pour des problèmes de santé, ne peut momentanément plus assurer la transformation de ses produits. Un planning est mis en place pour que les amapiens se relaient à l'atelier «moulage de beurre » pour le remplacer.

- Le maraîcher de l'AMAP L'Aneth a besoin d'aide pour nettoyer ses planches de cultures et remonter ses serres, mises à mal par la tempête. Une douzaine d'amapiens se mobilisent. En un week-end, une dizaine de planches de cultures sont nettoyées et trois serres de $500 \mathrm{~m}^{2}$ sont montées. 


\section{- Une proximité relationnelle étonnamment faible dans les trois circuits}

Enfin, la proximité relationnelle est étonnamment faible dans les trois circuits $\left(M_{P V C}=\right.$ 2,39, $M_{A M A P}=2,97, M_{\text {marché }}=2,72$ ). Malgré les contacts possibles entre producteurs et consommateurs - constituant un élément fondateur du caractère alternatif de ces démarches (Holloway et alii, 2007 ; Jarosz, 2000) -, la réalité des échanges semble plus mitigée. Ce résultat fait écho à certains travaux portant sur le lien producteurconsommateur en vente directe (Dubuisson-Quellier et LeVelly, 2008 ; Lamine, 2008) qui invitent à ne pas idéaliser cette relation. Certains consommateurs voient en effet dans ces circuits des modalités d'achat avantageuses (livraison à domicile, qualité des produits, etc.) mais ne souhaitent pas forcément rencontrer les producteurs. La plupart ne consacrent du temps à l'échange avec les producteurs que ponctuellement, lorsqu'ils recherchent de l'information concernant la qualité du produit.

Si aucune différence n'existe entre les PVC et les marchés, la proximité relationnelle est toutefois plus forte dans les AMAP (estimation de contraste ${ }_{A M A P-P V C}=0,726 ; p<$ 0,001, $\left.{ }_{\text {AMAP-marché }}=0,904 ; p<0,001\right)$. Ces écarts peuvent s'expliquer par le cadre et le contenu des échanges proposés. Ils s'inscrivent en effet principalement sur le lieu de la transaction marchande pour les producteurs vendant en marché et en PVC, alors que les AMAP stimulent les rencontres en dehors du domaine marchand (encadré 4). Elles ouvrent un champ relationnel plus large, dépassant l'échange autour du produit ou des processus de production et rendant l'interconnaissance entre les producteurs et consommateurs possible. Des échanges conviviaux sont également favorisés par le prépaiement des paniers, permettant de sortir de la relation marchande au moment de la livraison des produits.

Ainsi, le pouvoir discriminant de l'outil de diagnostic de la proximité perçue est démontré: les formats de vente directe peuvent être différenciés sur la base des dimensions identifiées, à l'exception de la proximité d'accès. L'analyse comparée des scores de proximité permet par ailleurs d'identifier des leviers d'action pour les producteurs qui souhaitent développer la proximité perçue dans leurs points de vente. 


\section{Comment créer de la proximité : recommandations managériales}

De façon générale, sur la base des analyses réalisées, deux types de recommandations peuvent être formulées. Les premières concernent les types de proximité à développer. Le modèle structurel testé montre qu'aucune d'entre elles ne doit être négligée si l'on souhaite agir sur la confiance à l'égard du circuit, certaines ayant toutefois plus d'impact que d'autres. La proximité identitaire est celle qui influence le plus la confiance, suivie de la proximité relationnelle, de processus et d'accès. Un zoom sur la proximité relationnelle semble intéressant pour deux raisons : (a) elle est relativement faible dans les trois circuits étudiés - alors qu'elle constitue l'un des fondements de la vente directe - et (b) elle n'influence pas directement la confiance. Néanmoins, son influence n'est pas négligeable : la proximité relationnelle avec le producteur, qu'elle soit liée à des échanges d'informations sur les produits ou à des échanges plus amicaux, a un impact positif sur la perception qu'ont les consommateurs des manières de faire (proximité de processus) et des valeurs défendues (proximité identitaire).

Les secondes recommandations concernent les moyens à mettre en œuvre pour développer les proximités identitaire, de processus et relationnelle. Nous proposons deux pistes de réflexion permettant aux circuits de vente directe de les accroitre : l'une sur la place à donner aux producteurs et l'autre sur l'appropriation d'outils marketing classiques permettant à chaque point de vente de véhiculer ses valeurs et principes d'actions.

\section{Interroger la place du producteur dans la vente directe}

Les circuits de vente directe de produits alimentaires doivent faire face à une concurrence qui s'intensifie. De nouvelles formes de distribution en circuits courts se développent, avec une présence très faible voire inexistante du producteur : les systèmes de vente de paniers par Internet avec intermédiaires, pour lesquels le producteur n'est pas présent, en sont un exemple (www.lespaniersdemartin.com, www.potagercity.fr ). La grande distribution cherche elle aussi à s'emparer de cette nouvelle valeur ajoutée apportée par l'image du producteur, à l'instar des Alliances Locales développées par E.Leclerc ou du Meilleur d'Ici de Casino. Face à cette concurrence, la présence du 
producteur sur le lieu de vente est souvent présentée comme un élément fort de différenciation (Merle et Piotrowski, 2012). La volonté de «mettre un visage derrière le produit », traduction possible du «teikei », ancêtre japonais de l'AMAP, renvoie à ce désir du consommateur de pouvoir entrer en contact avec les producteurs, qu'il le fasse réellement ou non. Assurer lui même la vente de ses produits présente toutefois une contrainte forte pour le producteur qui doit alors cumuler des tâches de production, de commercialisation et parfois de transformation. Deux visions s'opposent ainsi, l'une en faveur d'une relation directe toujours plus poussée, l'autre en faveur d'une relation centrée sur l'accessibilité du producteur et non sur son entière disponibilité.

A la question «le producteur doit-il être là tout le temps? », nos résultats donnent un argument pour répondre par la négative, si l'objectif de sa présence est de développer la proximité relationnelle. En effet, malgré leur présence permanente dans les trois circuits de vente directe étudiés, les scores de proximité relationnelle sont faibles. Néanmoins, les échanges avec le producteur, même s'ils sont ponctuels et peu chargés affectivement, influencent la proximité de processus et identitaire, et, indirectement la confiance. Des formats dans lesquels l'agriculteur serait là moins souvent mais autour d'évènements sur le lieu de vente ou sur le lieu d'exploitation, pourraient créer autant de proximité relationnelle et renforcer indirectement les deux autres formes de proximité. La co-construction de ces événements avec les consommateurs, pour les mettre en position d'acteurs, semble être également un levier d'action intéressant, comme le montrent les scores plus élevés de proximité relationnelle en AMAP. Il en est de même pour la co-construction des principes d'action, illustrée dans ce circuit par une participation des adhérents aux activités de production.

Nos résultats montrent par ailleurs que l'influence de la proximité relationnelle sur les proximités identitaire et de processus existe, mais qu'elle n'est pas si élevée qu'on pourrait le supposer pour des circuits de vente directe. En ce sens, ils interrogent sur la nécessité d'échanges forts avec les producteurs sur les lieux de vente directe. L'accessibilité du producteur, c'est-à-dire la facilité avec laquelle les consommateurs peuvent entrer en contact avec lui, pourrait être suffisante pour faire émerger de la confiance. Dès lors ce qui est important n'est pas tant le fait d'échanger régulièrement mais de donner au consommateur la possibilité de le faire (Prigent-Simonin et HéraultFournier, 2005). Des outils de communication qui rendent la présence du producteur «virtuelle», tout en maintenant la possibilité de contacts directs, peuvent être utilisés 
en parallèle pour accroitre proximités identitaire et de processus. Certains circuits de vente directe développent déjà des outils en ce sens (encadré 5).

\section{Encadré 5 : Des producteurs présents virtuellement}

En vente directe, l'image du producteur peut être utilisée sur les supports de communication afin de participer à la construction de proximités.

Le trombinoscope: une carte géographique, permettant de localiser les fermes et d'identifier par une photo chacun des producteurs commercialisant ses produits dans le magasin. Cet outil visuel permet d'associer le produit avec un territoire et son « fabricant ». Il est de plus en plus utilisé dans les PVC.

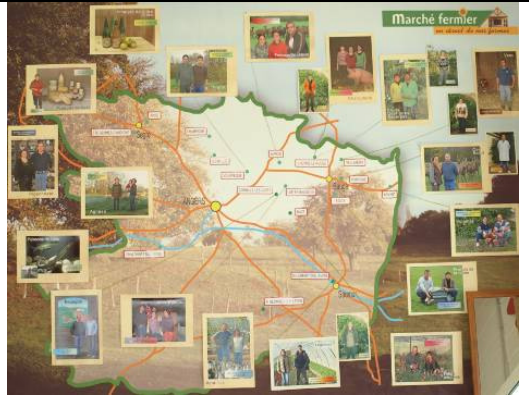

La photo de chaque producteur ou du collectif sur les outils de communication proposés : flyer, site Internet, carte de visite...
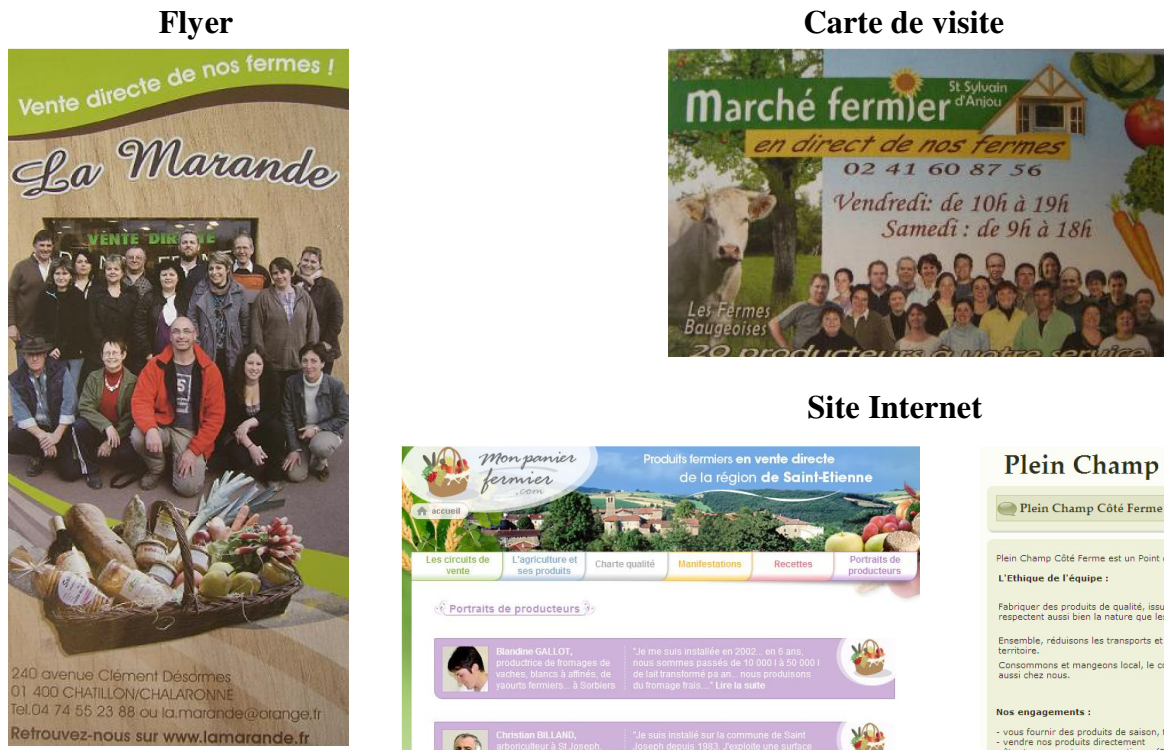

Site Internet

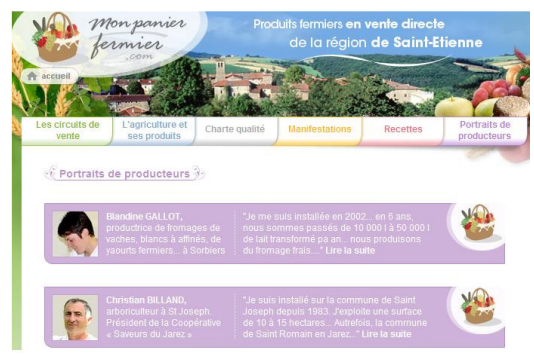

Plein Champ Cote Ferme

Plein Champ cat tone
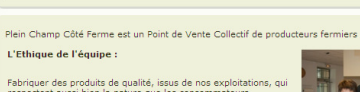

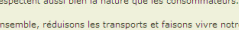
tatricie:

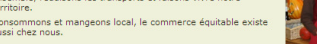
vos cenagocmentits:

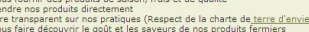

Un slogan évocateur : la présence des producteurs peut transparaître dans un slogan. A l'Ilot Fermier, PVC de Mayenne, le choix a été d'insister sur la présence des producteurs en magasin notamment en précisant sur ses flyers : "Des producteurs pour vous servir». Les consommateurs n'avaient en effet pas réalisé qu'ils avaient à faire aux producteurs.

\section{S’approprier les outils classiques du marketing}

L'analyse des différences de proximité perçue par circuit montre par ailleurs qu'il reste essentiel, même pour les circuits de vente directe, de «travailler sur des basiques », 
c'est-à-dire formaliser et communiquer leurs valeurs (accroissement de la proximité identitaire) et principes d'action concernant à la fois les modes de production, de transformation et de commercialisation (proximité de processus).

Les définir collectivement et montrer qu'ils sont partagés par tous les producteurs donne des repères aux consommateurs facilitant l'identification de similarités avec l'organisation (Palmatier et alii, 2006). La formalisation de cette expression collective peut être réalisée au travers de chartes, spécifiques à une initiative ou fédérant plusieurs d'entre elles. Pour véhiculer ces valeurs et principes, la création d'une identité visuelle, d'une marque et de supports informatifs, outils « classiques » du marketing, apparait dès lors pertinente. Elle permettrait de pallier en partie la faiblesse des interactions avec les producteurs. Relativement simples, ces outils constituent toutefois des éléments importants à mettre en œuvre pour favoriser les proximités identitaire et de processus dans des circuits de vente directe. Les rencontres avec les producteurs sur le lieu de vente, tant mises en avant, semblent en effet ne pas suffire, comme le montrent les faibles scores de proximité relationnelle.

Le tableau 2 présente certains outils en ce sens. S'ils s'adressent à tout type de circuit de vente directe, ils doivent être appliqués par chaque point de vente de façon singulière, en accord avec les principes et valeurs défendus.

\section{Conclusion}

Cette recherche montre ainsi l'intérêt managérial de la proximité dans le contexte de la vente directe de produits alimentaires. Les proximités relationnelle, identitaire et de processus jouent en effet un rôle dans le développement et le maintien de relations de long terme : elles permettent de donner confiance aux consommateurs en la capacité du circuit de vente directe à offrir la performance attendue ainsi qu'en l'intégrité de ces circuits. Se pose toutefois plus largement la question de la place et du rôle des différentes formes de proximité dans la séquence traditionnelle en marketing relationnel: satisfaction confiance - engagement (Morgan et Hunt, 1994). Cette interrogation mériterait d'être traitée dans de futures recherches. Si d'autres antécédents de la confiance étaient intégrés, le pourcentage de variance expliquée par les proximités pourrait être réduit. Par ailleurs, la proximité influence-t-elle la confiance pour tous les acheteurs en vente 
directe ? L'implication dans le circuit de distribution ou la sensibilité à la consommation responsable pourraient modérer la relation entre les proximités et la confiance.

Les proximités relationnelle, identitaire et de processus permettent par ailleurs de différencier des circuits de vente directe. Il serait intéressant de valider leur pouvoir discriminant par rapport à d'autres formes de circuits courts avec un intermédiaire ou à des circuits longs. De même, il parait pertinent de poursuivre les recherches portant sur la place du producteur dans la création de proximités selon trois axes. Le premier axe consisterait à étudier l'impact des outils de communication dans la création de proximités identitaire et de processus. Il serait possible de tester dans quelle mesure l'absence de proximité relationnelle avec le producteur peut être compensée par la mise en place d'outils «de médiation ». Le second axe aurait pour objet l'approfondissement de la notion de proximité relationnelle et de ses deux facettes potentielles - sociale et fonctionnelle. Enfin, un troisième axe pourrait étudier la délégation partielle de l'activité de vente à des vendeurs, qui interroge sur leur capacité à être perçus comme une source d'information aussi crédible et honnête que le producteur et à générer autant de proximité. 
Tableau 2

Accroitre la proximité identitaire et de processus en vente directe : un retour aux

« basiques » Id : identitaire, Proc. : processus

\begin{tabular}{|c|c|c|c|c|c|}
\hline & \multirow[t]{2}{*}{ Action } & \multirow{2}{*}{\multicolumn{2}{|c|}{ Exemples }} & & $\begin{array}{l}\text { le de } \\
\text { mité } \\
\text { illée }\end{array}$ \\
\hline & & & & Id. & Proc. \\
\hline \multirow{3}{*}{$\sum_{\substack{\infty \\
0}}^{\frac{1}{0}}$} & $\begin{array}{l}\text { Mettre en } \\
\text { place une } \\
\text { charte }\end{array}$ & \multicolumn{2}{|c|}{$\begin{array}{l}\text { - Comité des Produits Fermiers de la Loire: création d'une charte } \\
\text { éthique fermière autour de l'origine des produits et des modes de } \\
\text { production respectueux lenvironnement } \\
\text { (www.produitsfermiersloire.com). } \\
\text { - Association des producteurs fermiers du Rhône: charte qualité sur } \\
\text { l'accueil, l'origine fermière et la qualité des produits } \\
\text { (www.producteurs-fermiers-rhone.com). }\end{array}$} & $X$ & $X$ \\
\hline & $\begin{array}{l}\text { Rédiger des } \\
\text { fiches } \\
\text { techniques par } \\
\text { produit }\end{array}$ & \multicolumn{2}{|c|}{$\begin{array}{l}\text { - Cahier des charges défini pour chaque production précisant les } \\
\text { conditions d'élevage, de culture et de transformation } \\
\text { (www.produitsfermiersloire.com) } \\
\text { - Fiches techniques qui précisent les modalités de production pour } \\
\text { chaque produit les producteurs fermiers du Rhône }\end{array}$} & & $X$ \\
\hline & $\begin{array}{l}\text { S'engager } \\
\text { dans une } \\
\text { démarche de } \\
\text { garantie } \\
\text { participative }\end{array}$ & \multicolumn{2}{|c|}{$\begin{array}{l}\text { Démarche NESO: Naturel, Origine, Social, } \\
\text { Energie. Sur chaque axe de la boussole, une série } \\
\text { de critères invite les producteurs à réfléchir à leurs } \\
\text { pratiques, avec un groupe de consommateurs et } \\
\text { d'autres producteurs partenaires. Ces critères sont } \\
\text { inspirés des principes de l'agriculture biologique, } \\
\text { de Nature et Progrès et de la charte de } \\
\text { l'agriculture paysanne }\end{array}$} & $X$ & X \\
\hline \multirow{4}{*}{ 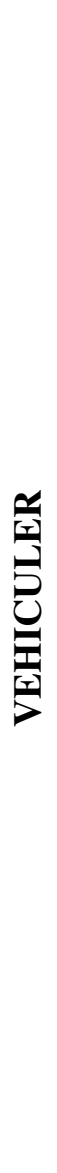 } & $\begin{array}{l}\text { Se doter d'une } \\
\text { identité } \\
\text { visuelle }\end{array}$ & \multicolumn{2}{|c|}{ 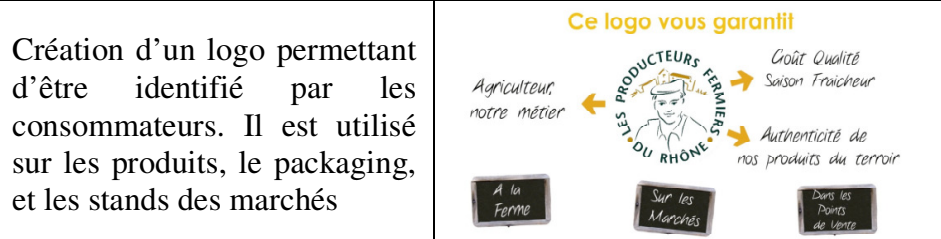 } & X & X \\
\hline & $\begin{array}{l}\text { Se doter d'une } \\
\text { marque }\end{array}$ & \multicolumn{2}{|c|}{$\begin{array}{l}\text { - Mis en place il y a } 30 \text { ans dans l'Aveyron, les } \\
\text { Marchés des Producteurs de Pays sont une } \\
\text { marque déposée par les Chambres } \\
\text { d'Agricultures. 2011: } 2000 \text { marchés dans } 33 \\
\text { départements } \\
\text { (http://www.marches-producteurs.com/) } \\
\text { - Contrairement à la pratique courante dans les } \\
\text { PVC, tous les produits du point de vente } \\
\text { Uniferme sont étiquetés sous la même marque }\end{array}$} & X & $X$ \\
\hline & \multirow{2}{*}{$\begin{array}{l}\text { Créer des } \\
\text { supports } \\
\text { informatifs } \\
\quad \text { sur les } \\
\text { engagements, } \\
\text { modes de } \\
\text { production et } \\
\text { les fermes }\end{array}$} & $\begin{array}{l}\text { Sur le marché paysan de la ville } \\
\text { de Grabels, créé en 2008, tous } \\
\text { les producteurs ont signé une } \\
\text { charte dans laquelle ils } \\
\text { s'engagent à indiquer clairement } \\
\text { sur leur étal et par l'intermédiaire } \\
\text { d'ardoises de couleurs l'origine } \\
\text { exacte de leurs produits. }\end{array}$ & $\begin{array}{l}\text { VERT PRODUCTION PERSONMELLE } \\
\text { ORANGE } \\
\text { MAUVE PRODUCTION D'UN } \\
\text { PRODUCTEUR VOISIN } \\
\text { (http/::WWW.ville-grabels.fr) }\end{array}$ & \multirow[t]{2}{*}{$X$} & \multirow[t]{2}{*}{$X$} \\
\hline & & $\begin{array}{l}\text { L'Ilot Fermier, PVC de } \\
\text { Mayenne, propose pour chaque } \\
\text { producteur impliqué une fiche } \\
\text { présentant le savoir-faire et les } \\
\text { productions de la ferme. }\end{array}$ & "Le cochon de la Colmont" & & \\
\hline
\end{tabular}




\section{Bibliographie}

Bellet M., Kirat T. et Largeron C. (1998), Approches multiformes de la proximité, Hermès Sciences Publication.

Bendapudi N. et Berry L. (1997), Customer's motivations for maintaining relationships with service providers, Journal of Retailing, 73, 12, 15-37.

Bergadaa M. et Del Bucchia C. (2009), La recherche de proximité par le client dans le secteur de la grande consommation alimentaire, Management et Avenir, 21, 121-135.

Bouba-Olga O. et Grossetti M. (2008), Socio-économie de proximité, Revue d'Economie Régionale et Urbaine, 3, 311-328

Brunori G. (2007), Local food and alternative food networks: a communication perspective, Anthropology of Food, 2, mars, http://aof.revues.org/index430.html.

Damperat M. (2006). Vers un renforcement de la proximité des relations client, Revue Française de Gestion, 32, 162, 115-125.

Dubuisson-Quellier et Lamine C. (2004), Faire le marché autrement. L'abonnement à un panier de fruits et de légumes comme forme d'engagement politique des consommateurs, Sciences de la Société, 62, mai, 145- 167.

Dubuisson-Quellier S. et Le Velly R. (2008), «Les circuits courts entre alternative et hybridation », dans Marechal G., Les circuits courts alimentaires, bien manger dans les territoires, Paris, Educagri, 105-112.

Filser M. et Vernette E. (2010), La proximité est-elle seulement une nouvelle mode du marketing ?, Décisions Marketing, 57, 5-7.

Gilly J.-P. et Torre A. (2000), Dynamiques de proximité, Paris, L'Harmattan.

Gurviez P. et Korchia M. (2002), Proposition d'une échelle de mesure multidimensionnelle de la confiance dans la marque, Recherche et Applications en Marketing, 17, 3, 41-61.

Hérault-Fournier C., Merle A. et Prigent-Simonin A.H. (2012), Comment les consommateurs perçoivent-ils la proximité à l'égard d'un circuit court alimentaire ?, Management \& Avenir, 53, 16-33.

Holloway L., Kneeafsey M., Venn L., Cox R., Dowler E. et Tuomainen H. (2007), Possible food economies : a methodological framework for exploring food productionconsomption relationships, Sociologica Ruralis, 47, 1, 1-17.

Jarosz L. (2000), Understanding agri-food networks as social relations, Agriculture and human values, 17, 279-283.

Lamine C. (2008), Les AMAP: un nouveau pacte entre producteurs et consommateurs?, Y. Michel, Paris. 
Merle A. et Piotrowski M. (2012), Consommer des produits alimentaires locaux : comment et pourquoi ?, Décisions Marketing, 67, 37-48.

Morgan R.M. et Hunt S.D. (1994), The Commitment-Trust Theory of Relationship Marketing , Journal of Marketing, 58, juillet, 20-38.

Palmatier R.W., Dant R.P., Grewal D. et Evans K.R. (2006), Factors influencing the effectiveness of relationship marketing: A meta analysis, Journal of Marketing, 70, octobre, 136-153.

Prigent-Simonin A.H. et Hérault-Fournier C. (2005), The role of trust in the perception of the quality of local food products with particular reference to direct relationships between producer to consumer. Anthropology of Food, 4, mai 2005

Rallet A. et Torre A. (2005), Proximity and localization, Regional Studies, 39, 1, 47-60.

Seyfang G. (2006), Ecological citizenship and sustainable consumption: Examining local organic food networks, Journal of Rural Studies, 22, 383-395.

Sirieix L., Grolleau G. et Schaer B. (2008), Do consumers care about food miles? An empirical analysis in France, International Journal of Consumer Studies, 32, 508-515.

Sirieix L. et Dubois P.-L. (1999), Vers un modèle qualité-satisfaction intégrant la confiance ?, Recherche et Applications en Marketing, 14, 3, 1-22

Vandercammen M. (2010), Quels sont les critères d'achat des consommateurs en 2010 ?, Rapport du CRIOC, Bruxelles. 


\section{Annexe 1 : Types de proximité et verbatim associés selon les formats de vente directe étudiés}

\begin{tabular}{|c|c|c|c|c|}
\hline & Définition & Circuit & Verbatim & Fréq. \\
\hline \multirow{3}{*}{ 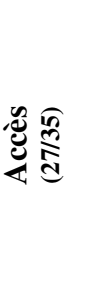 } & \multirow{3}{*}{$\begin{array}{l}\text { Facilité et praticité pour le } \\
\text { consommateur pour se rendre sur le } \\
\text { lieu de distribution (en termes de } \\
\text { distance, de temps, mais aussi de } \\
\text { commodité d'accès). }\end{array}$} & AMAP & $\begin{array}{l}\text { «Et celle de St Roch elle était proche de chez nous aussi », «à moi, c'était le côté distance que je puisse y aller à } \\
\text { pied » }\end{array}$ & $7 / 9$ \\
\hline & & Marchés & «accessibilité facile parce que j’habite à côté », « parce que il est près de chez nous et voilà il est facile d'accès » & $6 / 8$ \\
\hline & & PVC & $\begin{array}{l}\text { «Je trouve qu'il est bien situé parce que pour amener mes enfants à différents endroits j’y passe » « je trouve qu'il } \\
\text { est bien placé aussi, moi j'y vais à pied », « Je n'ai pas envie de prendre ma voiture pour aller acheter à manger » }\end{array}$ & $14 / 18$ \\
\hline \multirow{3}{*}{ 氖 } & \multirow{3}{*}{$\begin{array}{l}\text { Partage de valeurs avec l'initiative de } \\
\text { distribution en vente directe, } \\
\text { concernant les manières de produire } \\
\text { et de consommer }\end{array}$} & AMAP & $\begin{array}{l}\text { «La proximité est pas seulement avec les producteurs mais on se rend bien compte que la plupart des } \\
\text { consommateurs on se retrouve très souvent dans différentes manifestations on voit bien que la philosophie est } \\
\text { quand même partagée sur beaucoup de plans et beaucoup de points » }\end{array}$ & $7 / 9$ \\
\hline & & Marchés & $\begin{array}{l}\text { «C'est vrai même si je viens de milieu qui n'a rien à voir avec la terre je suis-je me sens toute proche d'eux », } \\
\text { «C'est vrai qu'il y a plein de valeurs sous-jacentes qui sont là quand on va au marché » }\end{array}$ & $6 / 8$ \\
\hline & & PVC & $\begin{array}{l}\text { «La philosophie c'est le développement durable, c'est peut-être un frein par rapport à notre société de } \\
\text { consommation qui est effrénée. » } \\
\text { «Je trouve que le bio, dans ce que je peux en percevoir, pour moi, c'est quand même une garantie d'avenir } \\
\text { durable pour la planète» }\end{array}$ & $17 / 18$ \\
\hline \multirow{3}{*}{ 莺 } & \multirow{3}{*}{ Lien direct crée avec le producteur } & AMAP & $\begin{array}{l}\text { «C'est un peu comme une vieille connaissance », «c'est de voir la personne qui a produit les fruits et légumes et } \\
\text { pouvoir échanger avec lui (...) avoir une autre relation qu'une relation commerciale bête et méchante» }\end{array}$ & $8 / 9$ \\
\hline & & Marchés & $\begin{array}{l}\text { «C'est un petit peu familier les rapports et puis il y a aussi quand on se connait bien », « mais être proche on peut } \\
\text { dire c'est acheter quelque chose et la personne vous reconnait », «c'est une relation conviviale cordiale amicale» }\end{array}$ & $7 / 8$ \\
\hline & & PVC & $\begin{array}{l}\text { «Ici il y a du contact, ce n'est pas anonyme (...) je demande des nouvelles de certains, il y a des habitudes, c'est } \\
\text { plus familiale, plus humain », «C'est amical, ce n'est pas de l'amitié, mais c'est amical » }\end{array}$ & $13 / 18$ \\
\hline \multirow{3}{*}{ 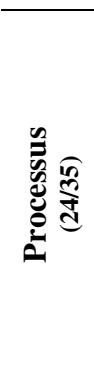 } & \multirow{3}{*}{$\begin{array}{l}\text { Partage de connaissances sur le } \\
\text { fonctionnement interne de l'initiative } \\
\text { de distribution en vente directe: } \\
\text { qualité et provenance des produits, } \\
\text { modes de préparation et de } \\
\text { distribution. }\end{array}$} & AMAP & $\begin{array}{l}\text { «On s'intéresse vraiment au produit et à sa production et au fonctionnement de l'exploitation dans son } \\
\text { ensemble », «Etre proche c'est-à-dire on a besoin de savoir d'où ça vient, savoir comment il fait, sa façon de } \\
\text { procéder sa technique » }\end{array}$ & $4 / 9$ \\
\hline & & Marchés & $\begin{array}{l}\text { «comment ils travaillent quelles sont les marches pour que le légume pousse toutes les étapes qu'il faut je ne sais } \\
\text { pas je leur demande je leur pose des questions », « vous savez comment ils cultivent comment ça se passe » }\end{array}$ & $6 / 8$ \\
\hline & & PVC & $\begin{array}{l}\text { «Ce que j'apprécie c'est la transparence donc ils ont affiché leur photo et ils ont quand même un discours qui est } \\
\text { assez transparent » } \\
\text { «C'est cher mais qu'on a des produits qui sont bons que c'est des petits producteurs qui ne mettent pas trop de } \\
\text { pesticides dans la terre, du moins j'espère» }\end{array}$ & $14 / 18$ \\
\hline
\end{tabular}




\section{Annexe 2 : Mesures utilisées : items et qualités psychométriques}

\section{Annexe 2.1. Items de mesure}

\section{Proximité d'accès}

PA1. Ce magasin/ marché/cette AMAP est très bien situé

$\mathrm{PA} 2$. Ce magasin/ marché/cette AMAP est très facile d'accès

PA3. Vous pouvez vous rendre très rapidement dans ce magasin/ marché/cette AMAP

PA4. Ce magasin/ marché/cette AMAP est très bien desservi

\section{Proximité identitaire}

PI1. Vous êtes en accord total avec les valeurs portées par ce magasin/ par les producteurs de ce marché/ par cette AMAP

PI2. Les valeurs de ce magasin/ des producteurs de ce marché/ de cette AMAP sont très importantes pour vous

PI3. Vos valeurs personnelles et celles de ce point de vente/ des producteurs de ce marché/ de cette AMAP sont très semblables

PI4. Vous partagez complètement la vision de l'agriculture prônée dans ce magasin/ par les producteurs de ce marché/ par cette AMAP

\section{Proximité de processus}

PP1. Dans ce magasin/ sur ce marché/ dans cette AMAP vous savez exactement comment sont fabriqués les produits de ces producteurs

PP2. Vous connaissez très bien les méthodes de production utilisées par les producteurs qui vendent dans ce magasin/ sur ce marché/ dans cette AMAP

$\mathrm{PP} 3$. Vous connaissez très bien leurs règles de fonctionnement et d'organisation

PP4. Vous savez très bien comment travaillent les producteurs qui vendent dans ce magasin/ sur ce marché/ dans cette AMAP

\section{Proximité relationnelle}

PR1. Vous avez des relations d'amitié avec les producteurs dans ce magasin/qui vendent sur ce marché/ de cette AMAP

PR2. Vous passez beaucoup de temps à échanger avec ces producteurs sur les produits

PR3.Vous passez beaucoup de temps à échanger avec ces producteurs sur d'autres thématiques que celles liées aux produits vendus

\section{Confiance}

C1. Ce magasin/ les producteurs de ce marché/ cette AMAP est très sincère vis-à-vis des adhérents/consommateurs

C2. Vous pensez que ce magasin/ les producteurs de ce marché/ cette AMAP cherche continuellement à apporter des réponses aux besoins des consommateurs

C3. Vous avez vraiment confiance en la qualité des produits de ce magasin/ des producteurs de ce marché/ cette AMAP

C4. Vous trouvez que ce magasin/ les producteurs auxquels vous achetez sur ce marché/ cette AMAP est/ sont très honnête(s) vis-à-vis de leurs clients/adhérents

C5. Pour vous, acheter des produits dans ce magasin /à des producteurs sur ce marché/ dans cette AMAP est une réelle garantie

C6. Les produits de ce magasin/des producteurs de ce marché/de cette AMAP vous apportent de la sécurité 
Annexe 2.2. Synthèse de la fiabilité et validité des échelles de mesure

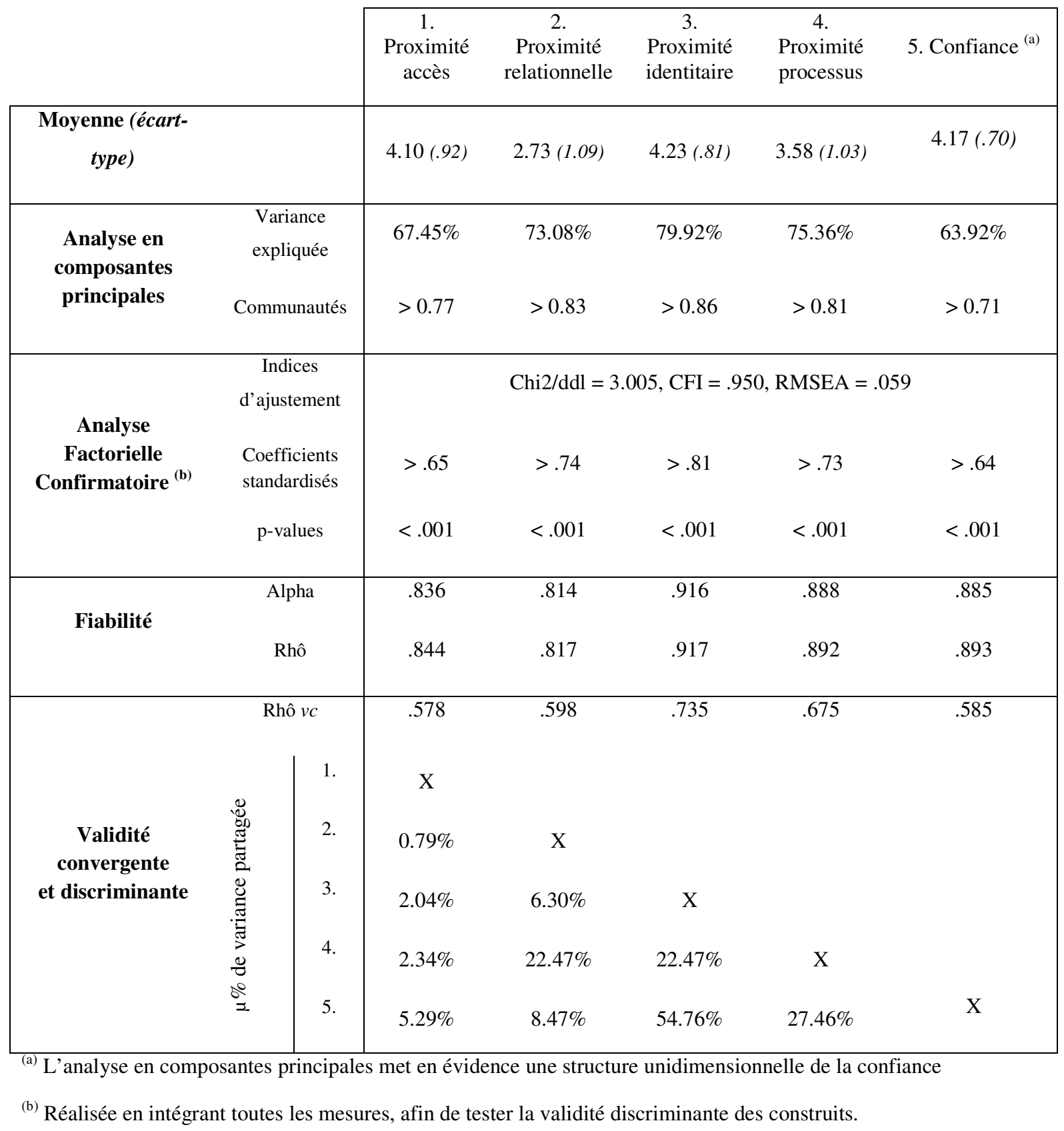

